\title{
Magnetic flux patterns in superconductors deposited on a lattice of magnetic dots: A magneto-optical imaging study
}

\author{
Diana G. Gheorghe and Rinke J. Wijngaarden \\ Department of Physics and Astronomy, Faculty of Sciences, Vrije Universiteit, De Boelelaan 1081, 1081 HV Amsterdam, The Netherlands
}

\author{
Werner Gillijns, Alejandro V. Silhanek, and Victor V. Moshchalkov \\ INPAC-Institute for Nanoscale Physics and Chemistry, K.U. Leuven, Celestijnenlaan 200D, B-3001 Leuven, Belgium
}

(Received 30 July 2007; revised manuscript received 12 October 2007; published 5 February 2008)

\begin{abstract}
We investigate the flux penetration in $\mathrm{Pb}$ films, of different shapes, deposited on top of a periodic array of $\mathrm{Co} / \mathrm{Pt}$ dots with perpendicular anisotropy by means of magnetization and magneto-optical measurements. A clear dependence of the critical current density on the magnetic state of the dots and their polarity with respect to the direction of the applied magnetic field is observed by both techniques. The magnetic state of the dots changes the flux penetration from smooth to channelling. Additionally, in the fully magnetized state, an anisotropic current distribution is observed in circular-shaped samples. The flux penetration is dominated by avalanches only for configurations which correspond to a high critical current, irrespective of its origin, be it low temperature, magnetization state of the dots, or angle between the lattice of dots and the edge of the sample.
\end{abstract}

DOI: 10.1103/PhysRevB.77.054502

PACS number(s): 74.78.-w, 74.78.Fk, 74.25.Dw

\section{INTRODUCTION}

In type-II superconductors submitted to an external magnetic field, flux penetrates in the form of vortices. Typically, their motion is prevented by pinning centers. Since vortices enter the sample through its borders, increasing the applied field results in a smooth distribution of vortices with a higher density at the boundary of the sample that progressively decreases towards its center. The resulting self-organized metastable state, known as the critical state, ${ }^{1}$ accounts for the irreversible magnetic response of type-II superconductors. In plain superconducting films the pinning, hence the maximum critical current $j_{c}$, is dominated by the strength of the intrinsic, randomly distributed defects. A more effective pinning (higher $j_{c}$ ) can be achieved by reducing the randomness in the spatial distribution of the pinning centers, as shown by studies carried out on superconducting films decorated with arrays of grooves, ${ }^{2}$ antidots (holes that fully thread the superconducting material), ${ }^{3}$ blind holes (holes that partially thread the superconducting material) ${ }^{4}$ or on superconductors evaporated on top of nonmagnetic dots. ${ }^{5-7}$ In such systems, the areas of reduced thickness act as effective pinning centers due to two pinning mechanisms: a reduction of the condensation energy due to the normal vortex core and electromagnetic pinning. ${ }^{8}$ Furthermore, the response of the superconductor in all the abovementioned structures is symmetric (irrespective of the polarity of the applied magnetic field).

A different situation emerges when a superconductor is in close vicinity to a magnetic dot lattice which induces vortexantivortex (V-AV) pairs in the superconducting layer. In the demagnetized state this dot structure mainly modulates the thickness of the superconducting layer and its effect is equivalent to that of blind holes or nonmagnetic dots (as discussed above). The periodicity of the magnetic dots array provides enhanced pinning properties, in particular, for an external field equivalent to a small integer number of vorti- ces per dot. If the dots are magnetized, however, the stray field is strong enough to thread the superconducting layer, generating vortices at the position of the dots and antivortices at interstitial positions. It follows naturally that for an infinite sample the number of vortices must be the same as the number of antivortices. At first sight the system can be considered still under zero average field and therefore no major differences on the magnetic response are expected. However, this approach is valid only if vortices and antivortices are fully equivalent, a condition that is realized with an array of in-plane magnetized micromagnets. In contrast to that, when using out-of-plane magnetized dots (the case we focus on this work) this symmetry is broken since vortices are constrained to intradot positions whereas antivortices sit in the interdot regions.

In this work we demonstrate that, as a result of the difference between vortices and antivortices induced by an underlying array of out-of-plane magnetized $\mathrm{Co} / \mathrm{Pt}$ dots in $\mathrm{Pb}$ films, the flux penetration is different for opposite field polarities. This result is confirmed by direct visualization of the flux penetration using magneto-optical imaging and can be qualitatively described within a critical state model involving two families of vortices. Additionally, we find that the morphology of the flux patterns is significantly influenced by the magnetization of the dots.

This paper is organized as follows. In Sec. II we describe the sample preparation and characteristics as well as the experimental techniques. Section III is dedicated to the magnetization measurements and Sec. IV provides a detailed description of the magneto-optical imaging experiments. Section V summarizes the results of our study.

\section{SAMPLE DETAILS AND EXPERIMENTAL TECHNIQUE}

The samples are 50-nm-thick $\mathrm{Pb}$ films evaporated by molecular beam epitaxy on top of a square array of $\mathrm{Co} / \mathrm{Pt}$ dots with out-of-plane magnetization. ${ }^{9,10}$ The $\mathrm{Pb}$ is separated from 
the magnetic dots by a $5 \mathrm{~nm}$ thick Ge buffer layer to prevent proximity effects. The dots consist of $2.5 \mathrm{~nm} \mathrm{Pt}+\mathrm{a}$ multilayer $[0.4 \mathrm{~nm} \mathrm{Co}+1.0 \mathrm{~nm} \mathrm{Pt}]_{10}$. Two sets of samples with different dot diameter $(1.0$ and $1.52 \mu \mathrm{m})$ but with the same periodicity of the square lattice of $2 \mu \mathrm{m}$ were prepared. Magnetization measurements at $T=5 \mathrm{~K}$ show that in both cases the dots are fully saturated at an applied field of $H_{\text {sat }}$ $=400 \mathrm{mT}$ and have a coercive field $H_{\text {coe }}=55 \mathrm{mT}$. Transport measurements indicate a superconducting transition temperature of $T_{c}=7.2 \mathrm{~K}$.

We label the samples according to their different sizes and geometries. Sample $S_{r}$ is a $0.4 \times 3 \mathrm{~mm}^{2}$ rectangle with $1 \mu \mathrm{m}$ diameter dots, sample $S_{s}$ is a square of $0.6 \times 0.6 \mathrm{~mm}^{2}$ with dots of $1.52 \mu \mathrm{m}$, sample $S_{d}$ is a square of $0.6 \times 0.6 \mathrm{~mm}^{2}$, rotated by $45^{\circ}$ with respect to the underlying magnetic lattice with dots of $1.52 \mu \mathrm{m}$, and sample $S_{c}$ is a circle of diameter $0.4 \mathrm{~mm}$ with dots of $1.52 \mu \mathrm{m}$.

Magnetization measurements were carried out in a commercial superconducting quantum interference device (SQUID) magnetometer MPMS-XL from Quantum Design with the applied field perpendicular to the sample plane. The magnetic flux distribution at the surface of the sample was mapped using the Faraday effect in a yttrium-iron garnet (YIG) film ${ }^{11}$ with a saturation field of $50 \mathrm{mT}$. The film was placed in close contact with the surface of the sample and the local magnetic field component, perpendicular to the plane of the sample, was determined by an improved polarization microscope setup: our magneto-optical image lock-in analyzer (MO-ILIA). ${ }^{12-14}$ The sample was placed inside a commercial Oxford Instruments vector magnet, the applied field was always perpendicular to the sample plane.

Prior to each experiment the dots were demagnetized, above $T_{c}$, by applying an oscillating magnetic field of decreasing amplitude, the value of the field at each semiperiod $N$ being given by $H_{\text {new }}^{(N)}=(-1)^{N} H_{\text {sat }} f^{N}$, with $f=0.9$. After this demagnetization, intermediate and full magnetization states of the dots were induced by applying magnetic fields in the range $\left[-H_{\text {sat }}, H_{\text {sat }}\right]$. In all experiments the initial state was prepared by cooling the samples in zero applied field. Subsequently, to prevent a change in the magnetization state of the $\mathrm{Co} / \mathrm{Pt}$ dots, magnetic fields much smaller that the coercive field $H_{\text {coe }}$ were applied.

\section{MAGNETIZATION MEASUREMENTS}

It was recently demonstrated ${ }^{15,16}$ that an underlying array of magnetic dots with perpendicular magnetization can stabilize in a nearby superconducting film a number $n$ of vortex-antivortex pairs determined by the geometrical details of the hybrid sample and the strength of the stray field emanating from the dots. Such vortex-antivortex configurations have been visualized ${ }^{16}$ by Hall probe microscopy. Adjusting the magnetic state of the dots allows one to change $n$ from $+n_{\max }$ to $-n_{\max }$ in steps of $\delta n=1$ in a fully reversible fashion. Recent studies showed that for the dots of $1 \mu \mathrm{m}$ diameter $+n_{\max }=3$, whereas for $1.52 \mu \mathrm{m}$ diameter $+n_{\max }=7 .{ }^{15}$ When the dots are in the demagnetized state or in the as-grown state, a balanced distribution of positive and negative domains within each dot coexists giving rise to little stray field.

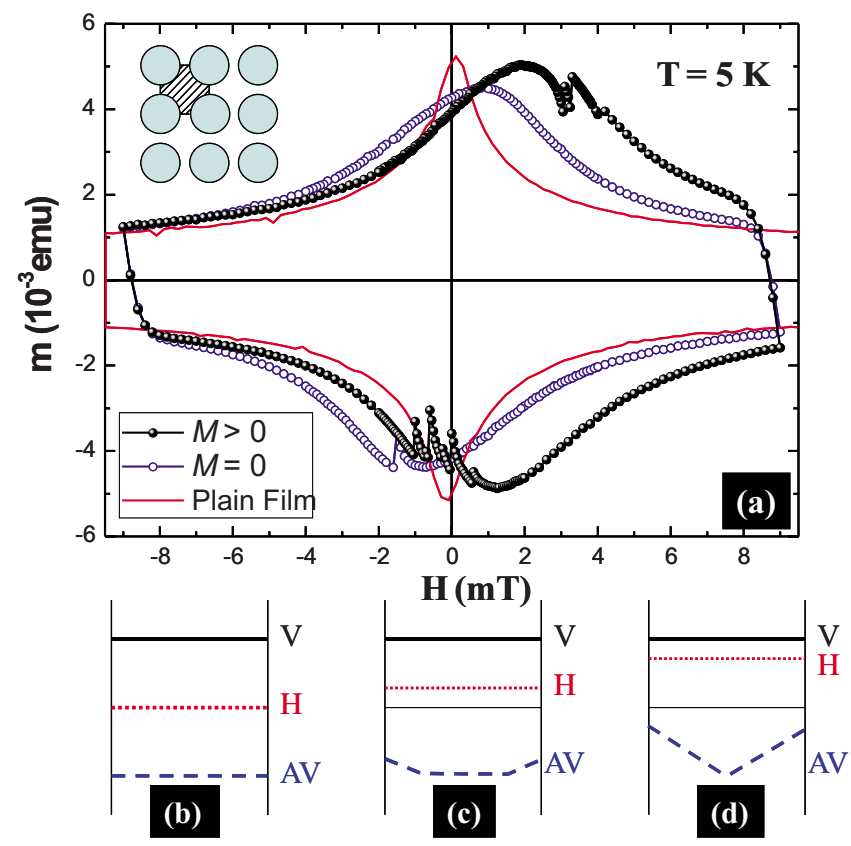

FIG. 1. (Color online) (a) Magnetization loops at $T=5 \mathrm{~K}$ of a superconducting $\mathrm{Pb}$ film covering an array of magnetic dots when the dots are demagnetized (open symbols) and fully magnetized in a positive field (full symbols). The magnetization response for the case $M<0$ is symmetric with the case $M>0$. The continuous line shows the hysteresis loop for a coevaporated plain $\mathrm{Pb}$ film. Inset (b) schematically shows the distribution of vortices (V) and antivortices (AV) after a zero field cooling. Insets (c) and (d) show the effect of removing antivortices as the external field $H$ is progressively increased.

Figure 1(a) shows the magnetization loops $M(H)$ of the hybrid sample $S_{r}$, dots of $1 \mu \mathrm{m}$, at $T=5 \mathrm{~K}$, when the dots are demagnetized (open symbols), fully magnetized (full symbols), and for a reference superconducting film without magnetic dots (full line). In general terms, the shape of magnetic hysteresis loops in superconducting materials can be explained by the critical state model where the depinning current of the superconductor $J c(H)$ is proportional to the width of the loop. A more rigorous analysis shows that the applied field $H$ is not the appropriate parameter to describe the field evolution of the magnetization $m$, but rather the local field $B$ felt by the superconductor. It has been previously reported $^{17,18}$ that it is indeed this discrepancy between $B$ and $H$ which accounts for the anomalous position of the low field peak, such as also observed in our Fig. 1(a). In general the total field experienced by the superconductor has a component generated by the screening currents that at remanence is commonly positive therefore giving rise to a maximum magnetization at slightly negative fields in the upper branch of the loop. However, in granular systems a different situation arises. Here it is the intergrain current which determines the self-field effects. In turn, the strength of these currents is intimately related with the actual field at the grain walls. Assuming that each grain can be modeled by a superconducting disk, it has been shown ${ }^{17}$ that at remanence the internal field $B$ at the grain walls is negative and therefore a compensating positive field is needed to optimize the intergrain cur- 
rent. Figure 1(a) shows that this effect is negligible in our $\mathrm{Pb}$ plain film but in the patterned film the peak is clearly shifted to the right in the upper branch. This indicates that there is little granularity in the original $\mathrm{Pb}$ films but as soon as the film is modulated by the presence of the dots a natural grain size determined by the separation between neighboring dots appears and consequently the peak in $m$ shifts towards positive fields. Interestingly a different behavior is observed when the dots are fully magnetized. First, the relative shift of the peaks on the upper and lower branches of the loop is substantially decreased, and secondly their average field position is no longer at $H=0$ but rather the whole loop is shifted towards positive fields, a feature that resembles the exchange-bias effect observed in antiferromagnetferromagnet bilayer systems. Notice that the effect we observe is nonsymmetric (i.e., occurs for both branches of the loop) and cannot be attributed to granularity. The observed magnetic bias of the superconducting loop arises from the lack of symmetry under field polarity switch of the applied field (of course, provided that the coercive field $H_{\text {coe }}$ $=400 \mathrm{mT}$ of the magnetic multilayers is not exceeded; clearly, for the range of fields used in our experiments, in Fig. 1 the applied magnetic field is smaller than $10 \mathrm{mT}$, this is satisfied).${ }^{19}$ Indeed, although V-AV are bounded pairs, vortices are strongly pinned on top of the $\mathrm{Co} / \mathrm{Pt}$ dots whereas antivortices occupy interstitial positions in between the dots and, provided that the stray magnetic fields emanating from the dots overlap, have a higher mobility. ${ }^{20}$ In the particular case shown in Fig. 1(a), $M>0$, at zero applied field, each unit cell contains three antivortices and the critical current is expected to be smaller than when no antivortices are present. In order to increase the critical current it is necessary to remove the antivortices from the interstitial positions. This can be done by introducing vortices with a positive external field which in turn annihilate the interstitial antivortices. Hence the shift of the whole $M>0$ curve to positive fields compared to the $M=0$ curve. The relative shift of the peaks in the hysteresis loops observed upon the change in the magnetization state of the $\mathrm{Co} / \mathrm{Pt}$ dots lattice can be explained as follows: in a good approximation we can assume that each cross-shaped interdots region acts as an individual grain connected to their four neighbors through the legs of the cross [see sketch in Fig. 1(a)]. When the dots are in the demagnetized state the complete interdots area is available for circulating the screening current and a maximum negative field is obtained at the cross' legs. Thus, a higher positive field is needed to compensate the field at that points. In contrast to that, when the dots are fully magnetized the effective area where intragrain currents can circulate shrinks and the effective field on the cross' legs decreases, therefore a smaller relative shift is obtained.

In fact, if one looks in more detail, the situation between vortices and antivortices in our system can be explained using a simple extension of the critical state model including two distinct families of vortices. This is schematically represented in Fig. 1(b) for $H=0$. On the one hand, there are vortices sitting on top of the dots which are nearly immobile (indicated by V). On the other hand, an equal number of interstitial antivortices (AV) give rise to an average field $B$ $=0$. As the field is increased [see panel (c) and (d) in Fig. 1] since the dots cannot trap extra vortices, incoming vortices occupy interstitial positions and annihilate antivortices. Essentially this is entirely similar to removing antivortices through the sample's border. The optimum critical current is achieved when, in average, the number of interstitial antivortices is minimum. In a thin film geometry with perpendicular field we can assume a macroscopically nearly flat distribution of vortices and therefore this maximum critical current should be close to, in our case, the perfect compensation field $H=3 H_{1}$, where $H_{1}=\phi_{0} / a^{2}$, is the first matching field, namely the externally applied magnetic field which generates one vortex per unit cell of the square array of magnetic dots with lattice parameter $a$ and $\phi_{0}$ the flux quantum. For our system $H_{1}=0.51 \mathrm{mT}$ and a shift of the whole hysteresis curve over $H=3 H_{1}=1.53 \mathrm{mT}$ is expected. This is in agreement with our observation of an average shift between the peaks of the hysteresis curves for the demagnetized and fully magnetized dots of $H \sim 1.6 \mathrm{mT}$, as shown in Fig. 1. (The magnetization response for the case $M<0$ is symmetric with the case $M>0$.)

In the present model, since the vortices on the dots are unable to move (i.e., are infinitely pinned) they are irrelevant for the irreversible magnetic response of the superconductor, in other words only the interstitial anivortices can build up a field gradient. From this point of view, panel (b) in Fig. 1 is somewhat similar to a field-cooling configuration with an internal field of $B=-3 H_{1}$.

\section{MAGNETO-OPTICAL RESULTS}

The properties of the samples were explored magnetooptically for several magnetic states of the $\mathrm{Co} / \mathrm{Pt}$ dots as well as different polarities of the applied magnetic field.

\section{A. Tunability of the critical current $j_{c}$}

In contrast to superconductors with artificial pinning sites introduced by irradiation or with nanoengineered antidots, where the pinning properties of the samples can not be modified once created, in superconductors decorated with magnetic dots the critical current $j_{c}$ can be continuously tuned by changing the magnetization of the magnetic template..$^{5,21-24}$ Most of these previous investigations have been performed at temperatures close to $T_{c}$ where the magnetic pinning dominates over the pinning produced by random defects, but little is known about the efficiency of the magnetic pinning at lower temperatures, deep into the superconducting state.

In order to fill this gap, we studied the flux penetration in a rectangular sample $S_{r}$ over a broad temperature range. Two different magnetic states of the dots and two orientations of the external field (parallel and antiparallel relative to the magnetic moment of the dots) were analyzed. Figure 2 summarizes the experimental results obtained at $T=2 \mathrm{~K}$ for the demagnetized and fully magnetized states. The most obvious feature of this figure is the pronounced difference of the flux penetration for different magnetic states of the dots. The largest flux penetration occurs when the magnetic moment $\mathbf{M}$ of the dots and the applied magnetic field $\mathbf{H}$ are antiparallel [Figs. 2(c) and 2(f)], whereas the lowest penetration 


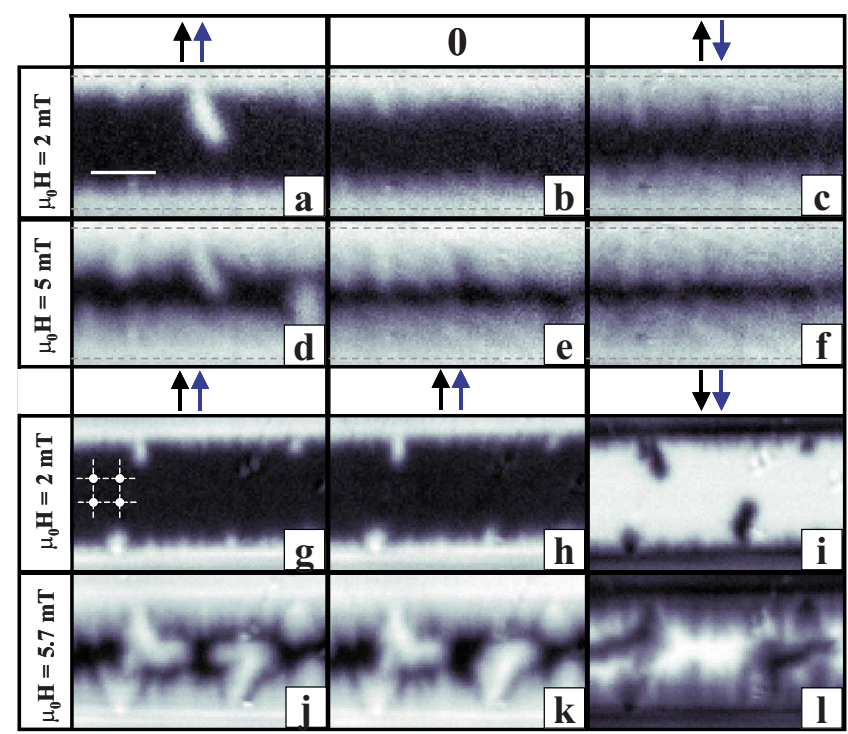

FIG. 2. (Color online) Magneto-optical images of the sample $S_{r}$, at $T=2 \mathrm{~K}$, for three different magnetization states of the $\mathrm{Co} / \mathrm{Pt}$ dots: demagnetized, panels (b) and (e); fully magnetized in a negative field, images (c) and (f); fully magnetized in a positive magnetic field, panels (a) and (d). Images (a), (b), and (c) show the sample in an applied external magnetic field of $2 \mathrm{mT}$ whereas in images (d), (e), and (f) the external field is $5 \mathrm{mT}$. Images (g), (h), (i), and (j), (k), (l) correspond to applied magnetic fields of 2 and $5.7 \mathrm{mT}$, respectively, and have been taken during three different experiments carried out under the same experimental conditions to show the degree of reproducibility of the avalanches. White corresponds to a high local magnetic field $H_{z}$ and black to $H_{z}=0$. The orientation of the $\mathrm{Co} / \mathrm{Pt}$ lattice, which is underneath the whole film, with respect to the superconducting strip is shown schematically (not to scale) in panel (g) by the white full dots. The scale bar corresponds to $200 \mu \mathrm{m}$.

takes place when $\mathbf{M}$ and $\mathbf{H}$ are parallel [Figs. 2(a) and 2(d)]. The demagnetized state, shown in Figs. 2(b) and 2(e), lies in between the previous two. These findings directly indicate that in the antiparallel configuration the critical current density reaches its lowest value, whereas in the parallel configuration $j_{c}$ is maximum. As we anticipated in the previous section, the observed $\mathbf{M}$-dependent penetration can be attributed to the fact that in the antiparallel configuration, the stray field of the magnetic lattice and the applied magnetic field add-up in the interstitial regions thus generating a higher local magnetic field in the sample. In contrast to that, in the parallel case stray field and applied field counteract each other leading to an enhancement of the critical current.

We now proceed to obtain values of $j_{c}$ from our magnetooptical images, which are a map of the out-of-plane component of the field $H_{z}$ on the surface of the sample. It has been shown ${ }^{25}$ that the magnetic field profiles at the surface of a thin film in the strip geometry are accurately described by $H_{z}(x)=H_{c} \operatorname{arctanh}\left[\left(x^{2}-b^{2}\right)^{1 / 2} / c|x|\right]$, for $b<|x|<a$, with $2 a$ the width of the sample, $2 b$ the width of the vortex free region of the sample, $c=\left(1-b^{2} / a^{2}\right)^{1 / 2}, H_{c}=j_{c} d / \pi$, and $d$ the sample thickness. The parameters $a, b$, and $d$ are known from the sample geometry and the magneto-optical images, therefore the fit of the experimental field profiles at the sur-

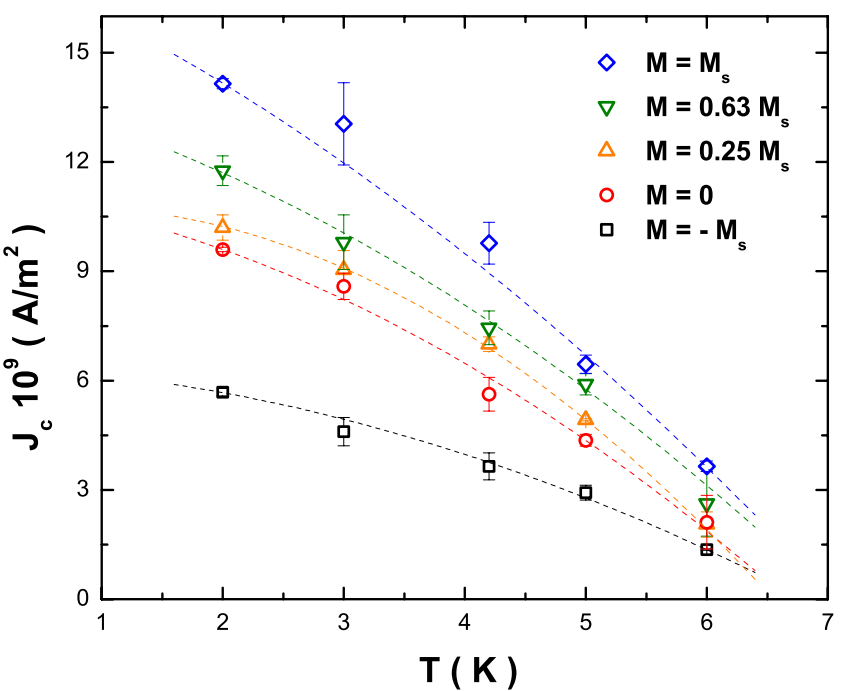

FIG. 3. (Color online) Temperature dependence of the critical current density $j_{c}$ for different magnetic states of the dots: demagnetized $(\bigcirc)$, fully magnetized parallel configuration $(\diamond)$, fully magnetized antiparallel configuration $(\square)$, partially magnetized parallel, $M=0.25 M_{s}(\triangle)$ and partially magnetized parallel, $M=0.63$ $M_{s}(\nabla)$. The error bars show the standard deviation as obtained from the fitting procedure (see text). The dashed lines are guides to the eye.

face of the sample yields directly the critical current density $j_{c}$. The result of this procedure is shown in Fig. 3 for several magnetic states. It is interesting to note that by changing the magnetic state of the dots the critical current of the system can be tuned by a factor of about 2.5 between the antiparallel and parallel configurations with fully magnetized dots.

\section{B. Flux front morphology}

Periodic lattices of pinning centers not only influence the pinning properties, ${ }^{4,26}$ but also modify the spatial distribution of the vortices as they penetrate into the sample, leading, for example, to channeling ${ }^{27-30}$ or anisotropic current distributions. ${ }^{31-33}$ Although the effect of the periodic array of pinning centers on the flux front morphology has been studied to some extent in superconductors patterned with antidots, similar investigations in superconductors decorated with magnetic lattices are scarce. ${ }^{34}$ In all cases, particular care must be paid to separate the influence of the orientation of the borders of the samples on the flux penetration from the effects associated with the periodic array of pinning sites. To address this issue we carried out experiments on three superconducting samples, each of them having different shape and orientation relative to the underlying magnetic lattice as specified in Sec. II.

In Fig. 4 we show magneto-optical images of samples $S_{s}$ and $S_{d}$ obtained at $T=6$ and $2 \mathrm{~K}$, for the fully magnetized and demagnetized states and for two polarities of the applied magnetic field (indicated by the arrows). A direct comparison of the images at $T=6 \mathrm{~K}$ in Figs. $4(\mathrm{~b}), 4(\mathrm{c}), 4\left(\mathrm{~d}_{1}\right), 4\left(\mathrm{~d}_{2}\right)$, and $4\left(d_{3}\right)$, reveals a rich variety of flux front morphologies. In the magnetized state, antiparallel configuration, Fig. 4(b), the 


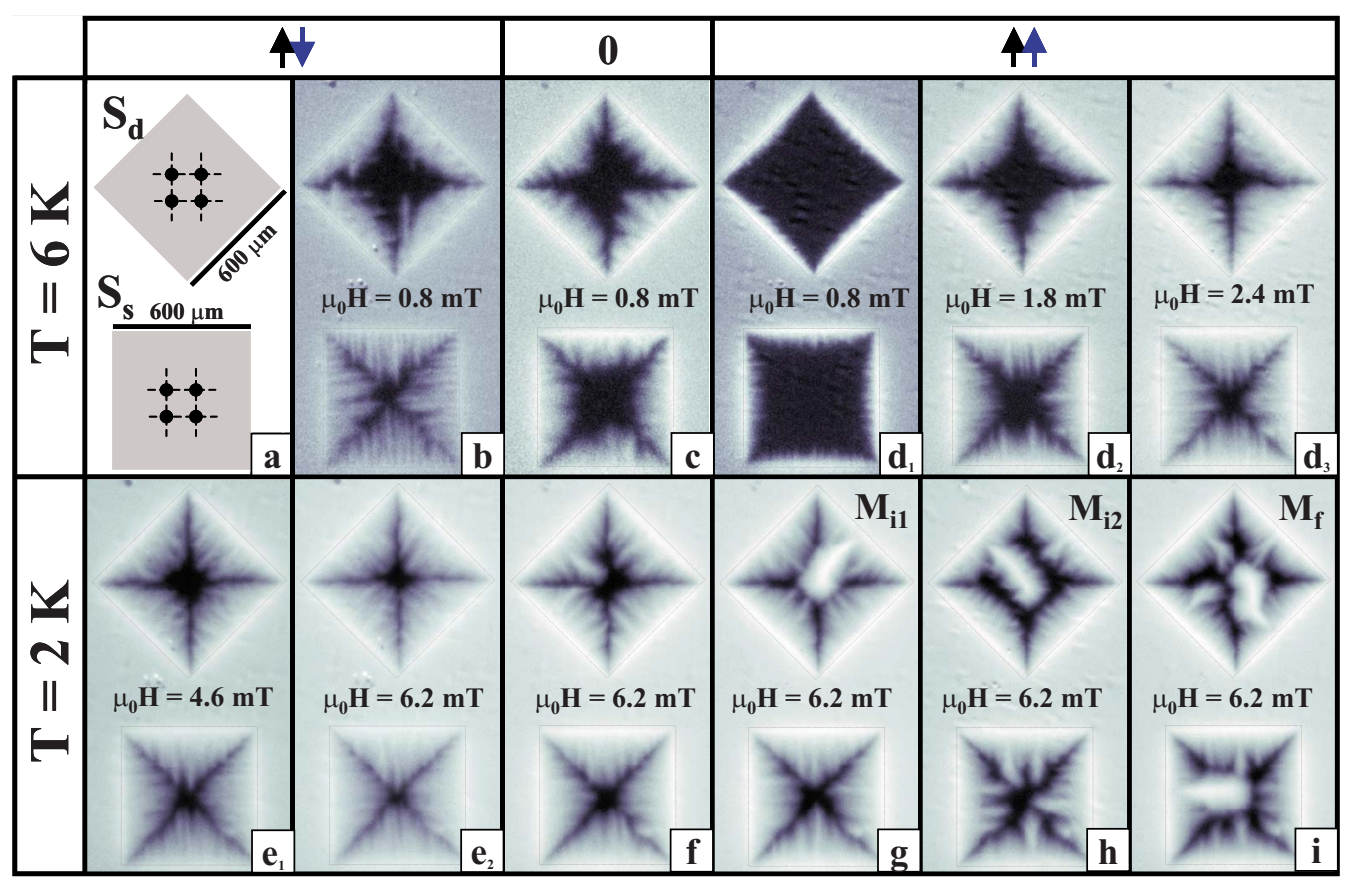

FIG. 4. (Color online) Magneto-optical images of the square $\left(S_{s}\right)$ and diamond $\left(S_{d}\right)$ samples at 6 and $2 \mathrm{~K}$. The orientation of the Co/Pt lattice with respect to the superconducting samples is shown schematically (not to scale) in panel (a). In all pictures, white corresponds to a high local magnetic field $H_{z}$ and black to $H_{z}=0$. The images show the magnetic flux distribution at the surface of the sample for several magnetization states of the $\mathrm{Co} / \mathrm{Pt}$ dots and polarities of the applied magnetic field: fully magnetized dots in the antiparallel [panels (b), ( $\left.\mathrm{e}_{1}\right)$, and $\left(\mathrm{e}_{2}\right)$ ] and parallel [panels $\left(\mathrm{d}_{1}\right),\left(\mathrm{d}_{2}\right),\left(\mathrm{d}_{3}\right)$, and (i), $M_{f}=M_{s}$ ] configurations, demagnetized dots [panels (c) and (f)], intermediately magnetized dots, $M_{i 1}=0.25 M_{s}$ [panel (g) $]$ and $M_{i 2}=0.63 M_{s}[$ panel (h)]. The values of the externally applied magnetic fields are indicated in each panel.

flux front exhibits clear channeling of vortices along the magnetic lattice vectors both for samples $S_{s}$ and $S_{d}$. By contrast, in the parallel configuration, Figs. $4\left(\mathrm{~d}_{1}\right), 4\left(\mathrm{~d}_{2}\right), 4\left(\mathrm{~d}_{3}\right)$, clear channels only appear in the square sample $S_{s}$.

We now discuss a tentative explanation for the dependence of the channeling on the magnetization state of the dots. In the magnetized state the magnetic field emanating from the $\mathrm{Co} / \mathrm{Pt}$ dots generates an equal amount of vortices at the position of the dots and antivortices (AV) at interstitial positions. These antivortices must remain in close vicinity to the magnetic dots ${ }^{35}$ and shield the magnetic field due to the dots. If a negative external magnetic field is applied (the antiparallel case), the entering antivotices experience the repelling forces of the AV already present in the system and, as a result, move along the center of interstitial channels parallel to the magnetic dot lattice vectors. Additionally, due to the repulsive interactions between $\mathrm{AV}$ and the incoming antivortices, some AV are pushed into adjacent channels. This leads to a blocking of the adjacent channel. Hence, due to any (thermal) fluctuation, flux penetration will take place along some interstitial channels, while other channels are blocked. This leads to the channeling observed in, e.g., Fig. 4(b). If, on the other hand, a positive external field is applied (the parallel case), the entering vortices annihilate with the antivortices nearby the dots. In this case the vortices simply flow around the dots and a smoother penetration takes place.

Lets now discuss the different flux front morphology in the square $S_{s}$ and diamond $S_{d}$ shown in Fig. 4. Since the Lorentz force is perpendicular to the sample's borders, for the square sample the vortices move along the lattice vectors (easy direction). For the diamond sample $S_{d}$, due to the $45^{\circ}$ rotation of the magnetic dot lattice with respect to the edge of the sample, the vortex motion is impeded. This results in a slower advancement of the flux quanta ${ }^{31,36}$ and a higher $j_{c}$.

Furthermore, by looking comparatively at the images shown in Figs. 4(b), 4(c), and 4(d $\left.\mathrm{d}_{1}\right)$, one can also observe, that for the same applied magnetic field, the distance penetrated by the magnetic flux is larger for the magnetized sample in the antiparallel configuration [Fig. 4(b)] than for the parallel configuration [Fig. $4\left(\mathrm{~d}_{1}\right)$ ]. This indicates that in the latter configuration, the critical current is higher, in agreement with our previous observations in the rectangular sample $S_{r}$. As shown by Figs. 4( $\left.\mathrm{e}_{2}\right), 4(\mathrm{f})$, and 4(i), this behavior persists also at low temperatures.

In contrast to the high-temperature behavior, at $T=2 \mathrm{~K}$ no well defined channels can be seen in the antiparallel configuration [Fig. $\left.4\left(\mathrm{e}_{1}\right)\right]$, but instead the flux penetrates in a somewhat more compact front. This difference is due to a stronger intrinsic (random) pinning at low temperature, thus reducing the relative importance of the dots on the pinning properties. Furthermore, in the demagnetized state [Fig. 4(f)] and in the intermediate [Fig. 4(g) and 4(h)] and full magnetization states [Fig. 4(i)], the flux fronts are distorted by avalanches resulting from thermomagnetic instabilities. ${ }^{37,38}$ Note that the avalanches are more prominent in the higher $j_{c}$ cases (higher magnetization, sample $S_{d}$ ).

We also explored the properties of circular-shaped samples, both patterned $\left(S_{c}\right)$ and plain films of the same 


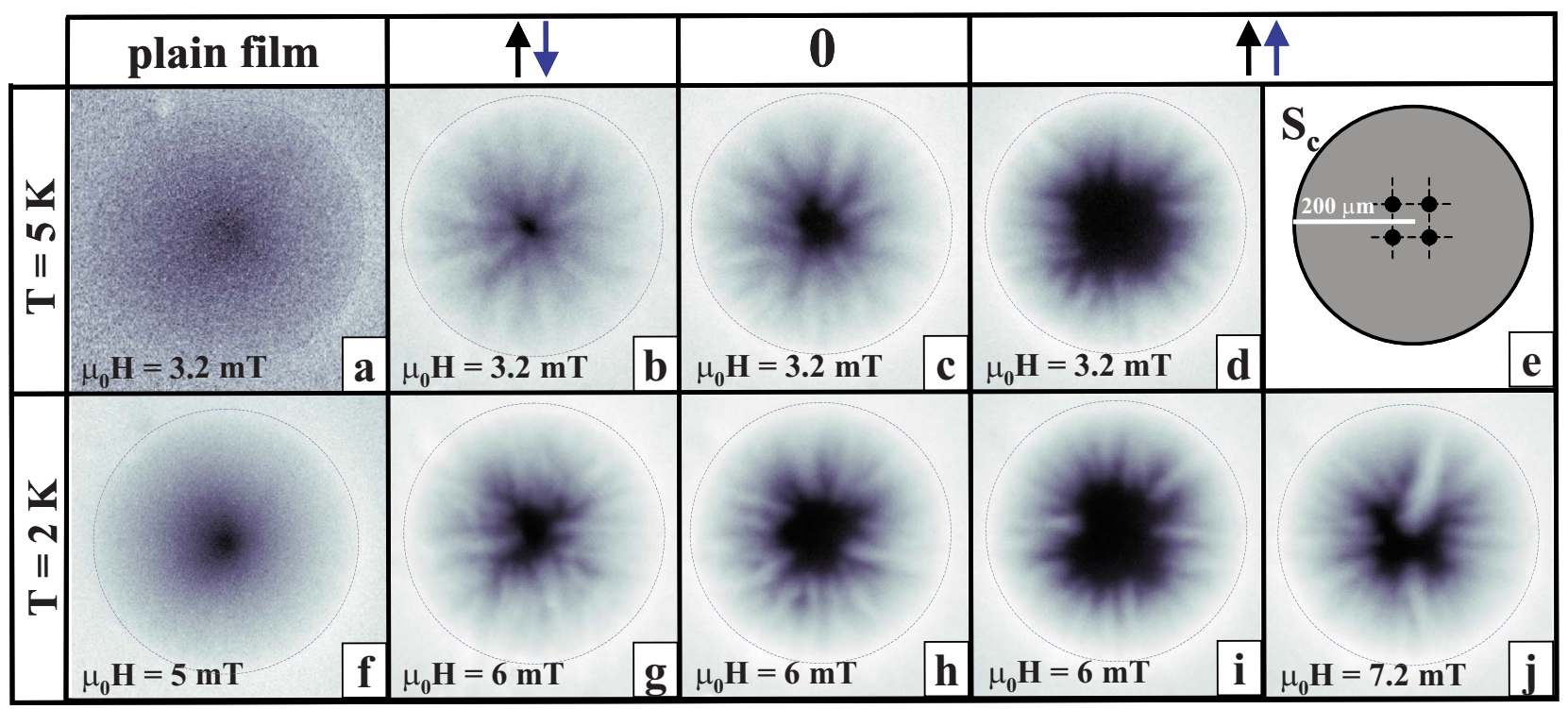

FIG. 5. (Color online) Magneto-optical images of a plain and decorated $\left(S_{c}\right)$ circular samples showing the magnetic field distribution at the surface of the sample at 2 and $5 \mathrm{~K}$. Images of the plain film are shown in panels (a) and (f), whereas the decorated sample $S_{c}$ is shown in panels (b), (g) (fully magnetized Co/Pt dots, antiparallel configuration); (c), (h) (demagnetized dots); (d), (i), and (j) (fully magnetized dots, parallel configuration). White corresponds to a high local magnetic field $H_{z}$ and black to $H_{z}=0$. The values of the applied magnetic fields are indicated in each panel. Panel (e) is a sketch of sample $S_{c}$; the orientation of the underlying magnetic lattice with respect to the sample is not drawn to scale.

thickness, see Fig. 5. In agreement with the results described above there is a clear difference between the demagnetized [Figs. 5(c) and 5(h)] and fully magnetized states in the parallel [Figs. 5(d) and 5(i)] and antiparallel configurations [Figs. 5(b) and 5(g)]. The parallel configuration corresponds to a state in which the critical current is highest; which at low temperatures is reflected in the nucleation of avalanches only for this configuration, as shown in Fig. 5(j) [note that Fig. 5(j) is at higher fields than Figs. 5(g)-5(i)]. Furthermore, in contrast to the plain film [Figs. 5(a) and 5(f)] the decorated sample has, irrespective of the experimental configuration, a higher critical current, reflected in a smaller flux penetration for the same applied fields. Additionally, the flux fronts are considerably rougher.

The effect of the lattice of dots on the anisotropy of the critical current was also probed experimentally. Any deviation of the flux front from a circular shape unambiguously reflects the influence of the underlying magnetic lattice. To quantify the effect of the underlying lattice on the morphology of the flux fronts, we calculated the position of the flux fronts $R$, relative to the center of the sample for $S_{c}$. We consider two images of the sample, obtained in experiments carried out at $T=6 \mathrm{~K}$, with demagnetized dots, Fig. 6(a), and fully magnetized dots, Fig. 6(b), which correspond to roughly the same amount of flux penetration for both cases. Each plot in Fig. 6(c) shows the position of the flux front averaged using the symmetry group of the square (superimposing copies of the image which are rotated by multiples of $\pi / 2$ and mirror images thereof). This smoothing procedure has been done, for both images in Fig. 6, to emphasize the differences between the morphology of the flux fronts in the case of demagnetized and fully magnetized dots. In the demagnetized case $(\bigcirc)$ a circular flux front is observed, indi- cating that in this state the dots do not have a considerable influence on the flux fronts. By contrast, in the fully magnetized state $(\boldsymbol{\square})$ the front exhibits a four-fold symmetry which reflects the geometry of the underlying magnetic lattice. The flux penetrates differently along different directions in the sample, indicated by angle $\theta$ [Fig. 6(b)]. More explicitly, the maximum amount of flux penetration is observed for $\theta$ $=n \pi / 2$, and the minimum amount along directions defined by $\theta=(2 n+1) \pi / 4$, where $n$ is an integer. Since there are no current sinks or sources in the sample, the current flowing in the sample is the same everywhere. Hence, the observed difference in the flux penetration reflects an anisotropic distribution of the critical current density, with high $j_{c}$ along the $\theta=(2 n+1) \pi / 4$ directions and small $j_{c}$ along the $\theta=n \pi / 2$ directions. ${ }^{31}$ At low temperatures the anisotropy is reduced, probably due to a stronger influence of the intrinsic pinning (which is due to randomly distributed defects). This is also clearly seen by comparing Fig. 5(d) with Fig. 6(b). Notice that a change in temperature of only one degree (from 5 to $6 \mathrm{~K})$ significantly enhances the anisotropy.

\section{Avalanches}

As already briefly mentioned in the previous subsections, at low temperatures the smooth flux penetration is accompanied by abrupt flux jumps or avalanches, as shown in Figs. 2(a), 2(d), and 2(g)-2(1); Figs. 4(f)-4(i); and Fig. 5(j). In all the experiments presented in this work the sweep rate of the external field was the same, namely $0.1 \mathrm{~T} / \mathrm{min}$. The flux jumps often nucleate at roughly the same positions in the sample, as seen most clearly in Figs. 2(g)-2(i), taken in three different experiments carried out under identical conditions. This indicates that the instabilities are triggered by static 


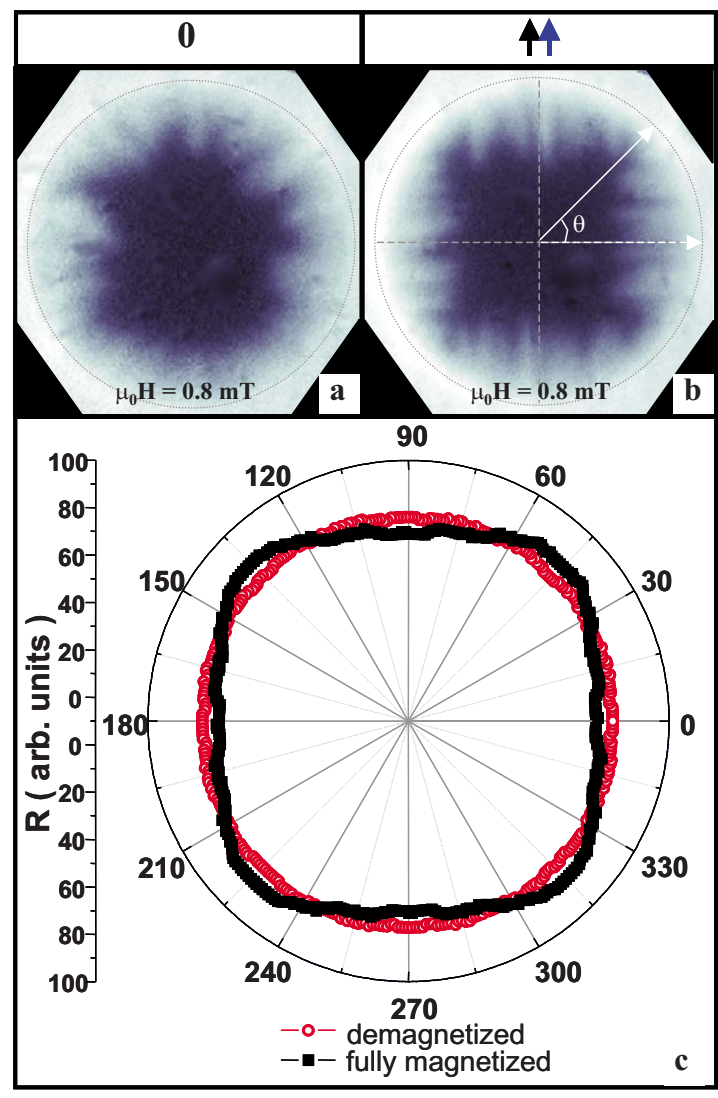

FIG. 6. (Color online) Magneto-optical images of the sample $S_{c}$ for the demagnetized (a) and magnetized (b) states of the dots and the associated positions of the magnetic flux fronts (averaged using the symmetry of the square, see text) relative to the center of the sample (c) for the demagnetized $(\bigcirc)$ and fully magnetized ( $\mathbf{\square})$ states. Both magneto-optical images were taken in experiments carried out at $T=6 \mathrm{~K}$. White corresponds to a high local magnetic field $H_{z}$ and black to $H_{z}=0$.

defects. Once an avalanche has nucleated, it remains frozen with increasing applied field until the critical slope builds up again, generating a new avalanche on top of an existing one, as shown in Figs. 2(j)-2(1).

In the rectangular sample $S_{r}$, Figs. 2(g)-2(1) and square sample $S_{s}$, Figs. 4(g)-4(i), these avalanches mainly proceed along the lattice vectors of the magnetic dot array, however, in the diamond $S_{d}$ in Figs. 4(f)-4(i), and circle $S_{c}$ in Fig. 5(j) the influence of the underlying lattice on the morphology of the flux jumps is much less pronounced.

In several theoretical studies, ${ }^{38,39}$ invoking a thermomagnetic origin of the instability, it was shown that higher critical currents promote the nucleation of avalanches. In agreement with these predictions we observed flux jumps only for the parallel configuration, i.e., the configuration corresponding to the highest $j_{c}$. They occur for fully as well as intermediately magnetized states, as shown in Figs. 4(g)-4(i). Each image corresponds to a different experiment in which the desired magnetization state of the dots was prepared by first demagnetizing the sample and subsequent application of magnetic fields of magnitudes $\mu_{0} H=125 \mathrm{mT}\left(M=0.25 M_{s}\right)$, $\mu_{0} H=215 \mathrm{mT}\left(M=0.63 M_{s}\right)$, and $\mu_{0} H=400 \mathrm{mT}\left(M=M_{s}\right)$ at
$T>T_{c}$. The value of the saturation magnetization $M_{s}$ and the relation $M(H)$ were determined from the hysteresis curve of the magnetic lattice, measured prior to the evaporation of the superconducting $\mathrm{Pb}$ film. Interestingly, in the diamondshaped sample $S_{d}$, where $j_{c}$ is high, due to the $45^{\circ}$ angle between dot lattice and edge, avalanches systematically occur also in the demagnetized state [Fig. 4(f)].

Irrespective of the size of the dots underlying the $\mathrm{Pb}$ film, avalanches were only observed at and below $2 \mathrm{~K}$. However, the lower threshold field for which the avalanches occur $H_{\mathrm{av}}$ depends on the size of the magnetic dots. In sample $S_{r}$ (dots of $1 \mu \mathrm{m}$ ) flux jumps already appear at applied magnetic fields of $\mu_{0} H_{\mathrm{av}}=1.4 \mathrm{mT}$, whereas in samples $S_{s}, S_{d}$, and $S_{c}$ (dots of $1.52 \mu \mathrm{m}$ ) avalanches nucleate typically at fields around $4 \mathrm{mT}$ (see also below). This is because, in the fully magnetized state, the interdot stray field is bigger for dots of $1.52 \mu \mathrm{m}$ diameter and hence the applied magnetic fields necessary to fully compensate it is bigger.

Minor differences between the applied fields at which avalanches nucleate in the samples with dots of $1.52 \mu \mathrm{m}$ were observed, depending upon the geometry of the superconductor. Thus, in the circular sample $S_{c}$, avalanches start to develop at an applied field of $\mu_{0} H_{\mathrm{av}}=5 \mathrm{mT}(+/-0.1 \mathrm{mT})$, in the diamond-shaped $S_{d}$ at $\mu_{0} H_{\mathrm{av}}=4 \mathrm{mT} \quad(+/-0.1 \mathrm{mT})$, whereas in the square $S_{s}$ at $\mu_{0} H_{\mathrm{av}}=3.8 \mathrm{mT}(+/-0.1 \mathrm{mT})$. Also, $H_{\mathrm{av}}$ was larger for higher magnetization states of the $\mathrm{Co} / \mathrm{Pt}$ dots (hence the change in $H_{\mathrm{av}}$ is mainly due to the different compensating field and not to changes in $j_{c}$ ).

\section{CONCLUSION}

We have shown that in superconductors patterned with a lattice of magnetic dots the critical current can be tuned practically at will over a broad range of temperatures by tuning the magnetization state of the dots.

The magnetic flux penetration displays a rich morphology characterized for $S_{d}$ by compact flux fronts in the parallel magnetized state of the dots and channelling in the antiparallel magnetized state. Additionally, in the parallel configuration the critical current is clearly anisotropic and the morphology of the flux fronts reflects the geometry of the underlying magnetic lattice.

We systematically find that avalanches are favored in samples with higher $j_{c}$, consistent with models ${ }^{38,39}$ based on a thermomagnetic instability. This holds irrespective of the origin of high $j_{c}$ be it overall sample shape, low temperature or field alignment (the parallel case).

\section{ACKNOWLEDGMENTS}

The authors are grateful to Mariela Menghini for suggestions. This work was supported by FOM (Stichting voor Fundamenteel Onderzoek der Materie) which is financially supported by NWO (Nederlandse Organisatie voor Wetenschappelijk Onderzoek), the K.U. Leuven Research Fund GOA/2004/2 program, the Belgian IAP, the Fund for Scientific Research-Flanders (F.W.O.-Vlaanderen), and the ESFNES Programmes. 
${ }^{1}$ C. Bean, Rev. Mod. Phys. 36, 31 (1964).

${ }^{2}$ O. Daldini, P. Martinoli, J. L. Olson, and G. Berner, Phys. Rev. Lett. 32, 218 (1974).

${ }^{3}$ V. V. Moshchalkov, M. Baert, V. V. Metlushko, E. Rosseel, M. J. Van Bael, K. Temst, R. Jonckheere, and Y. Bruynseraede, Phys. Rev. B 54, 7385 (1996); Phys. Rev. B 57, 3615 (1998).

${ }^{4}$ S. Raedts, A. V. Silhanek, M. J. Van Bael, and V. V. Moshchalkov, Phys. Rev. B 70, 024509 (2004).

${ }^{5}$ Y. Jaccard, J. I. Martin, M.-C. Cyrille, M. Velez, J. L. Vicent, and I. K. Schuller, Phys. Rev. B 58, 8232 (1998).

${ }^{6}$ A. Hoffmann, P. Prieto, and I. K. Schuller, Phys. Rev. B 61, 6958 (2000).

${ }^{7}$ M. Van Bael, Ph.D. thesis, Katholieke Universiteit Leuven, Belgium, 1998.

${ }^{8}$ A. Buzdin and D. Feinberg, Physica C 265, 303 (1996).

${ }^{9}$ W. B. Zeper, F. J. A. M. Greidanus, P. F. Carcia, and C. R. Fincher, J. Appl. Phys. 65, 4971 (1989).

${ }^{10}$ Z. G. Li and P. F. Carcia, J. Appl. Phys. 71, 842 (1992).

${ }^{11}$ L. A. Dorosinskii, M. V. Indenbom, V. I. Nikitenko, Yu. A. Ossip'yan, A. A. Polyanskii, and V. K. Vlasko-Vlasov, Physica C 203, 149 (1992).

${ }^{12}$ M. R. Koblischka and R. J. Wijngaarden, Semicond. Sci. Technol. 8, 199 (1995).

${ }^{13}$ R. J. Wijngaarden, K. Heeck, M. Welling, R. Limburg, M. Pannetier, K. van Zetten, V. L. G. Roorda, and A. R. Voorwinden, Rev. Sci. Instrum. 72, 2661 (2001).

${ }^{14}$ R. J. Wijngaarden, C. M. Aegerter, M. S. Welling, and K. Heeck, in Magneto-optical Imaging, edited by T. H. Johansen and D. V. Shantsev, Vol. 142 of Nato Science Series II (Kluwer Academic, Dordrecht, 2003), p. 61.

${ }^{15}$ A. V. Silhanek, W. Gillijns, M. V. Milosevic, A. Volodin, V. V. Moshchalkov, and F. M. Peeters, Phys. Rev. B 76, 100502(R) (2007).

${ }^{16}$ J. S. Neal, M. V. Milosevic, S. J. Bending, A. Potenza, L. San Emeterio, and C. H. Marrows, Phys. Rev. Lett. 99, 127001 (2007).

${ }^{17}$ M. R. Koblischka, L. Pust, A. Galkin, and P. Nálevka, Appl. Phys. Lett. 70, 514 (1996).

${ }^{18}$ M. Däumling and W. Goldacker, Z. Phys. B: Condens. Matter 102, 331 (1997).

${ }^{19}$ W. H. Meiklejohn and C. P. Bean, Phys. Rev. 102, 1413 (1956); 105, 904 (1957).

${ }^{20}$ M. Lange, M. J. Van Bael, A. V. Silhanek, and V. V. Moshchalkov, Phys. Rev. B 72, 052507 (2005).

${ }^{21}$ D. J. Morgan and J. B. Ketterson, Phys. Rev. Lett. 80, 3614
(1998).

${ }^{22}$ O. Geoffroy, D. Givord, Y. Otani, B. Pannetier, and F. Ossart, J. Magn. Magn. Mater. 121, 223 (1993).

${ }^{23}$ M. J. Van Bael, L. Van Look, K. Temst, M. Lange, J. Bekaert, U. May, G. Guntherodt, V. V. Moshchalkov, and Y. Bruynseraede, Physica C 332, 12 (2000).

${ }^{24}$ M. Lange, M. J. Van Bael, Y. Bruynseraede, and V. V. Moshchalkov, Phys. Rev. Lett. 90, 197006 (2003).

${ }^{25}$ E. H. Brandt, Rep. Prog. Phys. 58, 1465 (1995).

${ }^{26}$ A. V. Silhanek, S. Raedts, M. Lange, and V. V. Moshchalkov, Phys. Rev. B 67, 064502 (2003).

${ }^{27}$ A. Crisan, A. Pross, D. Cole, S. J. Bending, R. Wördenweber, P. Lahl, and E. H. Brandt, Phys. Rev. B 71, 144504 (2005).

${ }^{28}$ R. Surdeanu, R. J. Wijngaarden, E. Visser, J. M. Huijbregtse, J. H. Rector, B. Dam, and R. Griessen, Phys. Rev. Lett. 83, 2054 (1999).

${ }^{29}$ K. Harada, O. Kamimura, H. Kasai, T. Matsuda, A. Tonomura, and V. V. Moshchalkov, Science 274, 1167 (1996).

${ }^{30}$ S. Kolesnik, V. Vlasko-Vlasov, U. Welp, G. W. Crabtree, T. T. Piotrowski, J. Wrobel, A. Klimov, P. Przyslupski, T. Skoskiewicz, and B. Dabrowski, Physica C 341, 1093 (2000).

${ }^{31}$ M. Pannetier, R. J. Wijngaarden, I. Floan, J. Rector, B. Dam, R. Griessen, P. Lahl, and R. Wördenweber, Phys. Rev. B 67, 212501 (2003).

${ }^{32}$ M. S. Welling, R. J. Wijngaarden, C. M. Aegerter, R. Wördenweber, and P. Lahl, Physica C 404, 410 (2004).

${ }^{33}$ D. G. Gheorghe, M. Menghini, R. J. Wijngaarden, S. Raedts, A. V. Silhanek, and V. V. Moshchalkov, Physica C 437, 69 (2006).

${ }^{34}$ Y. Nozaki, Y. Otani, K. Runge, H. Miyajima, B. Pannetier, J. P. Nozières, and G. Fillion, J. Appl. Phys. 79, 8571 (1996).

${ }^{35}$ S. Erdin, Physica C 391, 140 (2003).

${ }^{36}$ A. V. Silhanek, L. Van Look, S. Raedts, R. Jonckheere, and V. V. Moshchalkov, Phys. Rev. B 68, 214504 (2003).

${ }^{37}$ T. H. Johansen, M. Baziljevich, D. V. Shantsev, P. E. Goa, Y. M. Galperin, W. N. Kang, H. J. Kim, E. M. Choi, M. -S. Kim, and S. I. Lee, Europhys. Lett. 59, 599 (2002); M. Baziljevich, A. V. Bobyl, D. V. Shantsev, E. Altshuler, and T. H. Johansen, Physica C 369, 93 (2002); E. Altshuler and T. H. Johansen, Rev. Mod. Phys. 76, 471 (2004).

${ }^{38}$ I. S. Aranson, A. Gurevich, M. S. Welling, R. J. Wijngaarden, V. K. Vlasko-Vlasov, V. M. Vinokur, and U. Welp, Phys. Rev. Lett. 94, 037002 (2005).

${ }^{39}$ D. V. Shantsev, A. V. Bobyl, Y. M. Galperin, T. H. Johansen, and S. I. Lee, Phys. Rev. B 72, 024541 (2005). 